\title{
THE STATE-SPACE OF THE LATTICE OF ORTHOGONALLY CLOSED SUBSPACES
}

\author{
E. CHETCUTI and A. DVUREČENSKIJ* \\ Mathematical Institute Slovak Academy of Sciences Stefanikova, SK-81473 Bratislava \\ e-mail: chetcuti@mat.savba.sk,dvurecen@mat.savba.sk
}

(Received 8 September, 2004; accepted 29 September, 2004)

\begin{abstract}
The notion of a strongly dense inner product space is introduced and it is shown that, for such an incomplete space $S$ (in particular, for each incomplete hyperplane of a Hilbert space), the system $F(S)$ of all orthogonally closed subspaces of $S$ is not stateless, and the state-space of $F(S)$ is affinely homeomorphic to the face consisting of the free states on the projection lattice corresponding to the completion of $S$. The homeomorphism is determined by the extension of the states. In particular, when $S$ is complex, the state-space of $F(S)$ is affinely homeomorphic to the state-space of the Calkin algebra associated with $\bar{S}$.
\end{abstract}

2000 Mathematics Subject Classification. Primary 46C05, 46L30. Secondary $03 \mathrm{G} 12$.

1. Introduction and preliminaries. Let $S$ be an inner product space (real, complex or quaternion) with inner product $\langle.$, . . . For any subspace $A \subset S$, we let $\bar{A}$ denote its completion. A subspace $A$ of $S$ is called orthogonally closed if $A=A^{\perp \perp}$, where $A^{\perp}=$ $\{x \in S:\langle x, y\rangle=0$ for all $y \in A\}$. Let us denote by $F(S)$ the set of all orthogonally closed subspaces of $S$. If we order $F(S)$ by the inclusion relation and endow it with the orthocomplementation $\perp$, then $F(S)$ becomes a complete orthocomplemented lattice (see [12], [6]). In this paper, let us denote by $P(S)$ the set of finite dimensional subspaces of $S$. Observe that $P(S) \subseteq F(S)$. For any vector $x \in S$ we let $[x]=\operatorname{span}\{x\}$.

We recall that a poset $(L, \leq, \perp, 0,1)$ is said to be orthocomplemented if, for every $a, b \in L$, we have (i) $a^{\perp \perp}=a$; (ii) $a \leq b$ implies $b^{\perp} \leq a^{\perp}$; (iii) $a \vee a^{\perp}=1$; and (iv) $0^{\perp}=$ 1. In addition, we say that an orthocomplemented poset $(L, \leq, \perp, 0,1)$ is orthomodular if, for any $a, b \in L$ (i) $a \leq b^{\perp}$ implies that $a \vee b$ exists in $L$; and (ii) $a \leq b$ implies that $b=a \vee\left(a^{\perp} \wedge b\right)$. Adopting the terminology taken from quantum system axiomatics (see, for example, [15], [6], [9]), we shall define a state on an orthocomplemented lattice $(L, \leq, \perp, 0,1)$ to be any mapping $s: L \rightarrow[0,1]$ such that (i) $s(a \vee b)=s(a)+s(b)$ whenever $a, b \in L$ and $a \leq b^{\perp}$; and (ii) $s(1)=1$. A state $s$ is said to be $\sigma$-additive if condition (i) is satisfied for every countable sequence $\left\{a_{i}: i \in \mathbb{N}\right\}$ of pairwise orthogonal elements of $L$ such that their join exists in $L$. We shall denote by $\mathcal{S}(F(S))$ the set of all states on $F(S)$. Then $\mathcal{S}\left(F(S)\right.$ ) is a convex compact subset of $\mathbb{R}^{F(S)}$. (Here we consider $\mathbb{R}^{F(S)}$ with the 'pointwise' topology.) Let $\mathcal{S}_{0}(F(S))$ denote the set of all free states on $F(S)$; i.e. the states that vanish identically on $P(S)$. Then $\mathcal{S}_{0}(F(S))$ is a face in $\mathcal{S}(F(S)$ ).

The completeness of an inner product space $S$, can be characterized by (i) the algebraic conditions on the lattice $F(S)$ (see [2]); and (ii) the measure-theoretic

*The authors acknowledge the support of the Grant VEGA No 2/3163/23 SAV and of APVT-51-032002, Bratislava, Slovakia. 
properties of $F(S)$ (see [10], [7], [3]). The Amemiya-Araki result asserts that whenever $F(S)$ is orthomodular, $S$ is complete. When $S$ is complete, $F(S)$ is merely the system of closed subspaces of $S$, and for any unit vector $x \in S$, the mapping $s_{x}: F(S) \rightarrow[0,1]$, $M \mapsto\left\|P_{M} x\right\|^{2}$ defines a $\sigma$-additive state (vector state) on $F(S)$. We recall that in this case the state-space $\mathcal{S}(F(S)$ ) coincides with the closure (in the pointwise topology) of the convex hull of $\left\{s_{x}: x \in S\right.$ and $\left.\|x\|=1\right\}$.

Let us now relax the assumption that $S$ is complete. J. Hamhalter and P. Pták proved [10] that a separable inner product space $S$ is complete if and only if $\mathcal{S}(F(S))$ contains at least one $\sigma$-additive state. This result was then generalized for nonseparable inner product spaces and signed-measures (see [6]). Furthermore, it can be shown (see [7]) that $S$ is complete if and only if there exists a unit vector $x \in \bar{S}$ such that the vector mapping $s_{x}: F(S) \rightarrow[0,1], M \mapsto\left\|P_{\bar{M}} x\right\|^{2}$ defines a state. Hence the state-space $\mathcal{S}(F(S))$ differs considerably from $\mathcal{S}(F(\bar{S}))$ when $S$ is incomplete. Indeed, one could have been somewhat justified to consider the system $F(S)$ - where $S$ is incomplete - as a possible candidate for a naturally born stateless orthocomplemented lattice.

In [4], the authors have exhibited a dense hyperplane $S$ of a separable Hilbert space $H$, satisfying $\mathcal{S}(F(S)) \neq \emptyset$. This gave an answer to the 'state on $F(S)$ problem' that was originally raised by Pták in [13]; see also [6, Problem 4.3.12]. It was also shown that, for each inner product space $S$ with a countable linear dimension, $F(S)$ admits states. The main objective of this paper is to describe the state-space $\mathcal{S}(F(S)$ ) for an important large class of incomplete inner product spaces. We prove that when $S$ is a strongly dense, incomplete space, every state in $\mathcal{S}(F(S))$ can be extended to a (unique) free state in $\mathcal{S}(F(\bar{S})$ ). In addition, if $S$ is complex, the state-space of $F(S)$ is affinely homeomorphic to the state-space of the Calkin algebra associated with the completion $\bar{S}$ of $S$.

As a by-product, a new orthomodular space is exhibited, the properties of which might be of interest and could be further investigated in connection to the foundations of quantum physics.

2. Results. An inner product space $S$, is said to be strongly dense in its completion $\bar{S}$, if every infinite dimensional closed subspace $A$ in $\bar{S}$ has a non-trivial intersection with $S$; i.e. $A \cap S \neq\{0\}$. Throughout this paper when we write ' $S$ is strongly dense' it should be always understood as ' $S$ is strongly dense in its completion'. We first justify ourselves for introducing the notion of 'strongly dense subspaces' by showing that in fact this class is 'large'.

Proposition 1. Let $S$ be an inner product space such that $\operatorname{dim} \bar{S} / S<2^{\aleph_{0}}$. Then $S$ is strongly dense in $\bar{S}$.

Proof. Suppose that $A \in F(\bar{S}), \operatorname{dim} A=\infty$ and $A \cap S=\{0\}$. If we let $U$ to be a Hamel (linear) basis of $A$, then it is clear that $\{u+S: u \in U\}$ is a linearly independent set in $\bar{S} / S$. Since $|U| \geq 2^{\aleph_{0}}$, it follows that $\operatorname{dim} \bar{S} / S \geq 2^{\aleph_{0}}$.

Observe that every incomplete hyperplane of a Hilbert space is strongly dense. The converse of Proposition 1 is not true; i.e. there are inner product spaces that are strongly dense and yet the co-dimension in their completion is $2^{\aleph_{0}}$. To see this fact, let $H$ be a separable Hilbert space, $\left\{e_{i}: i \in \mathbb{N}\right\}$ an orthonormal basis of $H$ and $\mathfrak{F}$, the collection of all infinite dimensional closed subspaces of $H$. Recall that $|\mathfrak{F}|=2^{\aleph_{0}}$. If we denote by $\omega$ the first ordinal with cardinality $2^{\aleph_{0}}$, we can express $\mathfrak{F}$ as $\left\{U_{\alpha}: \alpha<\omega\right\}$. 
Using transfinite induction, we can construct a linearly independent set of unit vectors $\bigcup_{\alpha<\omega}\left\{u_{\alpha}, v_{\alpha}\right\} \cup\left\{e_{i}: i \in \mathbb{N}\right\}$ in $H$ such that $u_{\alpha}, v_{\alpha} \in U_{\alpha}$, for each $\alpha<\omega$. Using Zorn's lemma, we can extend this set to a Hamel basis $K$ of $H$. Let $K^{\prime}=K \backslash\left\{u_{\alpha}: \alpha<\omega\right\}$, and define $S=\operatorname{span}\left\{K^{\prime}\right\}$. It is clear that $S$ is strongly dense in $H$ and $\operatorname{dim} H / S=2^{\aleph_{0}}$. If follows that the class of all strongly dense inner product spaces is relatively large; yet as the following proposition illustrates - not every inner product space is strongly dense.

Proposition 2. Let $S$ be an inner product space with a countable (infinite) linear dimension. Then $S$ is not strongly dense.

Proof. It is enough to exhibit one such inner product space since the inner product spaces with countable (infinite) linear dimension are unitarily equivalent.

Let $\left\{e_{i j}: i, j \in \mathbb{N}\right\}$ be an orthonormal basis of a separable Hilbert space $H$, and let $S=\operatorname{span}\left\{e_{i j}: i, j \in \mathbb{N}\right\}, v_{i}=\sum_{j=1}^{\infty} \frac{1}{2^{j}} e_{i j}$ and $A=\overline{\operatorname{span}}\left\{v_{i}: i \in \mathbb{N}\right\}$. Since $v_{i} \perp v_{k}$ for $i \neq k$, we have $\operatorname{dim} A=\infty$. It is easily seen that $A \cap S=\{0\}$; i.e. $S$ is not strongly dense.

Let $S$ be an inner product space. We shall define a binary relation on the elements of $F(S)$ as follows: $A \sim B$ if there exist finite dimensional subspaces $N_{1}, N_{2}$ of $S$ such that $A+N_{1}=B+N_{2}$. It is not difficult to check that $\sim$ is reflexive, symmetric and transitive; i.e. $\sim$ defines an equivalence relation on $F(S)$. Let $\mathfrak{F}(S)$ denote the partition $F(S) / \sim$. For any $A \in F(S)$, let $\langle A\rangle$ denote the equivalence class containing $A$. Then $\langle\{0\}\rangle$ and $\langle S\rangle$ represent the classes of finite and cofinite dimensional subspaces of $S$, respectively. For any $\langle A\rangle,\langle B\rangle \in \mathfrak{F}(S)$, we say that $\langle A\rangle$ is less than $\langle B\rangle$ and write $\langle A\rangle \leq\langle B\rangle$ if, for every $C \in\langle A\rangle$, there exists $D \in\langle B\rangle$ such that $C \subset D$. (Observe that $\langle A\rangle \leq\langle B\rangle$ if and only if there exists $C \in\langle A\rangle$ and $D \in\langle B\rangle$ such that $C \subset D$.) It is easy to show that $\leq$ is reflexive and transitive. Moreover, if $\langle A\rangle \leq\langle B\rangle$ and $\langle B\rangle \leq\langle A\rangle$, then we can find subspaces $C \in\langle A\rangle$ and $D \in\langle B\rangle$, such that $A \subset D \subset C$. Since $\operatorname{dim} C / A<\infty$, it follows that $A \sim D$, and therefore $D \in\langle A\rangle$. This implies that $\langle A\rangle=\langle B\rangle$; i.e. $\leq$ defines a partial order on $\mathfrak{F}(S)$. Indeed, in Proposition 3 , we prove that $\leq$ induces on $\mathfrak{F}(S)$ a lattice structure homomorphic to the lattice structure of $F(S)$. Let us first prove the following lemma.

Lemma 1. Let $A, B, C$ be subspaces of $S$ such that $A \subset B+M$ and $A \subset C+N$, where $M, N \in P(S)$. Then there exists $K \in P(S)$ such that $A \subset(B \cap C)+K$.

Proof. It is clear that we need to prove the statement only for the case when $\operatorname{dim} M=\operatorname{dim} N=1$; the rest would follow by a straightforward application of finite induction. We assume that $A \subset B+[m]$ and $A \subset C+[n]$, for some $m, n \in S$. Denote by $f$ and $g$ any two linear functionals on $B+[m]$ and $C+[n]$ respectively, such that $\operatorname{Ker}(f)=B$ and $\operatorname{Ker}(g)=C$. The kernel $\operatorname{Ker}\left(\left.f\right|_{A}\right)$ of the restriction of $f$ on $A$ is either $A$, or a hyperplane in $A$; we shall denote it by $F_{0}$. Of course, we have $F_{0} \subset B$ and $F_{0} \subset C+[n]$. If we let $F=\operatorname{Ker}\left(\left.g\right|_{F_{0}}\right)$, we have $F \subset B \cap C$ and $\operatorname{dim} A / F \leq$ 2. Thus $A=F+K$, for some finite dimensional subspace $K$ of $S$. Moreover, $A \subset(B \cap C)+K$.

Proposition 3. The partial ordering $\leq$ induces a lattice structure on $\mathfrak{F}(S)$ homomorphic to that of $F(S)$; i.e. for every $\langle A\rangle,\langle B\rangle \in \mathfrak{F}(S)$ we have

$$
\langle A\rangle \vee\langle B\rangle=\langle A \vee B\rangle \text { and }\langle A\rangle \wedge\langle B\rangle=\langle A \wedge B\rangle .
$$


Proof. It can easily be seen that $\langle A\rangle,\langle B\rangle \leq\langle A \vee B\rangle$. On the other hand, let $\langle D\rangle \in$ $\mathfrak{F}(S)$ such that $\langle A\rangle,\langle B\rangle \leq\langle D\rangle$. Then, there exist $D_{1}, D_{2} \in\langle D\rangle$ such that $A \subset D_{1}$ and $B \subset D_{2}$. Moreover, $D_{1}+M=D_{2}+N$, for some $M, N \in P(S)$. Let $C \in\langle A \vee B\rangle$; i.e. $C+M^{\prime}=(A \vee B)+N^{\prime}$, for some $M^{\prime}, N^{\prime} \in P(S)$. Thus,

$$
C \subset C+M^{\prime}=(A \vee B)+N^{\prime} \subset D_{1}+M+N^{\prime},
$$

and therefore $\langle A \vee B\rangle \leq\langle D\rangle$; i.e. $\langle A\rangle \vee\langle B\rangle=\langle A \vee B\rangle$.

On the other hand, it is clear that $\langle A \wedge B\rangle \leq\langle A\rangle$ and $\langle A \wedge B\rangle \leq\langle B\rangle$. Let $\langle D\rangle \in \mathfrak{F}(S)$ such that $\langle D\rangle \leq\langle A\rangle,\langle D\rangle \leq\langle B\rangle$ and let $C \in\langle D\rangle$. Then $C \subset A_{1}$ and $C \subset B_{1}$, for some $A_{1} \in\langle A\rangle$ and $B_{1} \in\langle B\rangle$. There exist $M_{1}, M_{2}, N_{1}, N_{2} \in P(S)$ such that

$$
C \subset A_{1} \subset A_{1}+M_{1}=A+N_{1} \text { and } C \subset B_{1} \subset B_{1}+M_{2}=B+N_{2} .
$$

By Lemma 1, it follows that $C \subset(A \wedge B)+K$, where $K \in P(S)$. This implies that $\langle D\rangle \leq\langle A \wedge B\rangle$, and therefore $\langle A\rangle \wedge\langle B\rangle=\langle A \wedge B\rangle$.

When $F(S)$ is orthomodular (and therefore, in view of [2], $S$ is complete) the system $\mathfrak{F}(S)$ can be equipped with a 'natural' orthocomplementation $\perp$. We prove this in the following proposition.

PROPOSITION 4. If $F(S)$ is orthomodular, then the mapping $\perp: \mathfrak{F}(S) \rightarrow \mathfrak{F}(S)$, $\langle A\rangle \mapsto\left\langle A^{\perp}\right\rangle$, defines an orthocomplementation. In this case, $(\mathfrak{F}(S), \leq, \perp,\langle\{0\}\rangle,\langle S\rangle)$ is an orthomodular lattice with the smallest and greatest elements being $\langle\{0\}\rangle$ and $\langle S\rangle$, respectively.

Proof. First we show that the mapping $\perp$ is well defined, i.e. we show that if $\langle A\rangle=\langle B\rangle$, then $\left\langle A^{\perp}\right\rangle=\left\langle B^{\perp}\right\rangle$. Indeed, let $N_{1}, N_{2} \in P(S)$ such that $A+N_{1}=B+N_{2}$. Let $M_{1}=A^{\perp} \wedge\left(A+N_{1}\right)$ and $M_{2}=B^{\perp} \wedge\left(B+N_{2}\right)$. Then $M_{1}$ and $M_{2}$ are finite dimensional and, in view of the orthomodular law, we have

$$
A+N_{1}=A \vee M_{1}=A \oplus M_{1} \text { and } B+N_{2}=B \vee M_{2}=B \oplus M_{2}
$$

Furthermore, we have $A^{\perp}=\left(A \oplus M_{1}\right)^{\perp} \oplus M_{1}$ and $B^{\perp}=\left(B \oplus M_{2}\right)^{\perp} \oplus M_{2}$. Thus

$$
\begin{aligned}
M_{2}+A^{\perp} & =\left(M_{2}+\left(A \oplus M_{1}\right)^{\perp}\right)+M_{1} \\
& =\left(M_{2} \oplus\left(B \oplus M_{2}\right)^{\perp}\right)+M_{1} \\
& =B^{\perp}+M_{1},
\end{aligned}
$$

and therefore $\left\langle A^{\perp}\right\rangle=\left\langle B^{\perp}\right\rangle$.

It is easy to verify that the mapping $\perp$ is one-to-one, maps $\mathfrak{F}(S)$ onto itself, and if $\langle A\rangle \leq\langle B\rangle$, then $\langle B\rangle^{\perp} \leq\langle A\rangle^{\perp}$. Furthermore, $\langle A\rangle^{\perp \perp}=\langle A\rangle,\langle A\rangle \vee\langle A\rangle^{\perp}=\langle S\rangle$ and $\langle A\rangle \wedge$ $\langle A\rangle^{\perp}=\{0\}$; i.e. $\perp$ defines an orthocomplementation on $\mathfrak{F}(S)$. The orthomodularity of $\mathfrak{F}(S)$ follows directly from the orthomodularity of $F(S)$. Indeed, if $\langle A\rangle \leq\langle B\rangle$, we have $A \subset D$, for some $D \in\langle B\rangle$. Since $F(S)$ is orthomodular, we have $D=A \vee\left(A^{\perp} \wedge D\right)$, which implies that $\langle B\rangle=\langle D\rangle=\langle A\rangle \vee\left(\langle A\rangle^{\perp} \wedge\langle B\rangle\right)$. This completes the proof.

Now we prove that, for inner product spaces that are strongly dense in their completion, the lattices $\mathfrak{F}(S)$ and $\mathfrak{F}(\bar{S})$ are isomorphic as orthocomplemented lattices. Roughly speaking, this means that, for such inner product spaces, $F(S)$ differs from $F(\bar{S})$ only with respect to finite dimensional subspaces. Consequently, every measure 
on $F(\bar{S})$ that is identically zero on the set of finite dimensional subspaces of $\bar{S}$ should induce a measure on $F(S)$.

THEOREM 1. Let $S$ be an inner product space that is strongly dense in its completion. Then $\mathfrak{F}(S)$ is an orthomodular lattice. Furthermore, $\mathfrak{F}(S)$ is isomorphic to $\mathfrak{F}(\bar{S})$ (as orthomodular lattices).

Proof. That $\mathfrak{F}(S)$ is a lattice was shown in Proposition 3. It is not difficult to verify that the mapping $\psi: \mathfrak{F}(S) \rightarrow \mathfrak{F}(\bar{S}),\langle A\rangle \mapsto\langle\bar{A}\rangle$ is well defined; i.e. if $\langle A\rangle=\langle B\rangle$, then $\langle\bar{A}\rangle=\langle\bar{B}\rangle$. Let us proceed by showing that $\psi$ is one-one and onto. Indeed, if $\psi(\langle A\rangle)=\psi(\langle B\rangle)$, for some $\langle A\rangle,\langle B\rangle \in \mathfrak{F}(S)$, then $\bar{A} \oplus M=\bar{B} \oplus N$, for some $M, N \in P(\bar{S})$. Let us verify that $\operatorname{dim}((\bar{A} \oplus M) \cap S) / A<\operatorname{dim} M$. Let us assume the contrary and let $\left\{\left(a_{i}+m_{i}\right) / A: i \in I\right\}$ be a linearly independent set of vectors in the space $((\bar{A} \oplus M) \cap S) / A$, where $\infty>|I|>\operatorname{dim} M$. Then there exists a set of scalars $\left\{\alpha_{i}: i \in I\right\}$ such that $\sum_{i \in I} \alpha_{i} m_{i}=0$ and not all $\alpha_{i}$ 's are zero. This implies that

$$
\begin{aligned}
& \sum_{i \in I} \alpha_{i}\left(a_{i}+m_{i}\right) / A \\
& =\left(\sum_{i \in I} \alpha_{i} a_{i}\right) / A+\left(\sum_{i \in I} \alpha_{i} m_{i}\right) / A \\
& =\left(\sum_{i \in I} \alpha_{i} a_{i}\right) / A=0,
\end{aligned}
$$

since $\sum_{i \in I} \alpha_{i} a_{i}+\sum_{i \in I} \alpha_{i} m_{i} \in S$ and hence $\sum_{i \in I} \alpha_{i} a_{i} \in \bar{A} \cap S=A$. Let $K \in P(S)$ be such that $(\bar{A} \oplus M) \cap S=A+K$. Using an argument as the previous one it is easy to show that $(\bar{B} \oplus N) \cap S=B+L$, for some $L \in P(S)$. Hence, we have $A+K=B+L$, and therefore $\langle A\rangle=\langle B\rangle$. For any $M \in F(\bar{S})$, let $M_{1}=\overline{(M \cap S)^{\perp \perp}}$ and $M_{0}=\overline{M \cap S}$. Since $S$ is strongly dense, we have $\left\langle M_{0}\right\rangle=\left\langle M_{1}\right\rangle=\langle M\rangle$, and therefore we have $\psi\left(\left\langle(M \cap S)^{\perp \perp}\right\rangle\right)=\langle M\rangle$; i.e. $\psi$ is onto. This means that we may (and in fact we shall) identify each element of $\mathfrak{F}(\bar{S})$ with $\langle\bar{A}\rangle$, for some $A \in F(S)$.

To show that $\psi$ is a lattice isomorphism it suffices to verify that $\langle\overline{A \vee B}\rangle=\langle\bar{A} \vee \bar{B}\rangle$ holds, for all $A, B \in F(S)$. Of course, we have $\bar{A} \vee \bar{B} \subset \overline{A \vee B}$ and therefore

$$
\overline{A \vee B}=(\bar{A} \vee \bar{B}) \oplus\left(\bar{A}^{\perp_{\bar{S}}} \wedge \bar{B}^{\perp_{\bar{S}}} \wedge(\overline{A \vee B})\right)
$$

If $\left(\bar{A}^{\perp} \bar{S} \wedge \bar{B}^{\perp_{\bar{S}}} \wedge(\overline{A \vee B})\right)$ is infinite dimensional, then it has a non-trivial intersection with $S$; i.e. there exists a non-zero vector $x \in(A \vee B) \wedge A^{\perp} \wedge B^{\perp}$; this is absurd. Hence $\langle\overline{A \vee B}\rangle=\langle\bar{A} \vee \bar{B}\rangle$.

We proceed further and show that the mapping $\perp: \mathfrak{F}(S) \rightarrow \mathfrak{F}(S)$, defined by $\langle A\rangle^{\perp}=\psi^{-1}\left(\psi(\langle A\rangle)^{\perp}\right)$, is an orthocomplementation on $\mathfrak{F}(S)$. That $\perp$ is well-defined on $\mathfrak{F}(S)$ follows directly from the definitions of $\psi, \psi^{-1}$ and the fact that $\perp$ is welldefined on $\mathfrak{F}(\bar{S})$. We prove that for every $A \in F(S),\langle A\rangle^{\perp}=\left\langle A^{\perp}\right\rangle$. By definition, $\langle A\rangle^{\perp}=\psi^{-1}\left(\left\langle\bar{A}^{\perp} \bar{S}\right\rangle\right)$. But $\overline{A^{\perp}} \in\left\langle\bar{A}^{\perp} \bar{S}\right\rangle$; indeed, if ${\overline{A^{\perp}}}^{\perp} \bar{S} \cap \bar{A}^{\perp} \bar{S}$ is infinite dimensional, then $A^{\perp \perp} \cap A^{\perp} \neq\{0\}$, which is false. Hence $\perp$ is an orthocomplementation on $\mathfrak{F}(S)$. This concludes our proof.

Observe that an inner product space $S$, is strongly dense if, and only if, the map $\langle A\rangle \mapsto\langle\bar{A}\rangle$ is a surjection from $\mathfrak{F}(S)$ onto $\mathfrak{F}(\bar{S})$. 
Lemma 2. Let $A \subset B \in F(S)$ and $s \in \mathcal{S}(F(S))$. Then $s(B)=s(A)+s\left(A^{\perp} \wedge B\right)$.

Proof. The assertion follows from the following equalities.

$$
\begin{aligned}
s(A)+s\left(A^{\perp} \wedge B\right) & =s(A)+s\left(\left(A \vee B^{\perp}\right)^{\perp}\right) \\
& =s(A)+1-s\left(A \vee B^{\perp}\right) \\
& =s(A)+1-s(A)-s\left(B^{\perp}\right) \\
& =1-s\left(B^{\perp}\right)=s(B) .
\end{aligned}
$$

Proposition 5. Suppose that $(\mathfrak{F}(S), \leq)$ admits an orthocomplementation $\perp$ such that $\langle A\rangle^{\perp}=\left\langle A^{\perp}\right\rangle$, for every $A \in F(S)$. There is an affine homeomorphism between the state-space of $\mathfrak{F}(S)$ and the face $\mathcal{S}_{0}(F(S))$ in the state-space of $F(S)$ consisting of all those states that vanish identically on the set of finite dimensional subspaces of $S$.

Proof. For each state $s$ on $F(S)$ vanishing on $P(S)$ we can define the mapping $\hat{s}$ : $\mathfrak{F}(S) \rightarrow[0,1]$, defined by $\hat{s}(\langle A\rangle)=s(A)$. Observe that the definition of $\hat{s}$ is independent of the representation of $\langle A\rangle$ because, in view of Lemma 2 , if $\langle A\rangle=\langle B\rangle$, then $s(A)=s(B)$. We shall now show that $\hat{s}$ defines a state on $\mathfrak{F}(S)$. Of course, we have $\hat{s}(\langle S\rangle)=1$. Let $\langle A\rangle,\langle B\rangle \in \mathfrak{F}(S)$ such that $\langle A\rangle \perp\langle B\rangle$; i.e. $\langle A\rangle \leq\langle B\rangle^{\perp}$. Then there exists $N \in P(S)$ such that $A \subset B^{\perp}+N=\left(B \wedge N^{\perp}\right)^{\perp}$. Since $B^{\perp}+N \in\left\langle B^{\perp}\right\rangle$, it follows that $B \wedge N^{\perp} \in\langle B\rangle$. Thus

$$
\begin{aligned}
\hat{s}(\langle A\rangle \vee\langle B\rangle) & =\hat{s}\left(\langle A\rangle \vee\left\langle B \wedge N^{\perp}\right\rangle\right) \\
& =\hat{s}\left(\left\langle A \vee\left(B \wedge N^{\perp}\right)\right\rangle\right) \\
& =s\left(A \vee\left(B \wedge N^{\perp}\right)\right) \\
& =\hat{s}(\langle A\rangle)+\hat{s}\left(\left\langle B \wedge N^{\perp}\right\rangle\right) \\
& =\hat{s}(\langle A\rangle)+\hat{s}(\langle B\rangle) ;
\end{aligned}
$$

i.e. $\hat{s}$ is a state on $\mathfrak{F}(S)$. It is very easy to check that the mapping^: $s \mapsto \hat{s}$ is one to one. We prove that it is also surjective. Let $w$ be a state on $\mathfrak{F}(S)$. Let us define $w_{0}: F(S) \rightarrow[0,1]$, $w_{0}(A)=w(\langle A\rangle)$. If $A \subset B^{\perp}$, then $\langle A\rangle \leq\langle B\rangle^{\perp}$, and hence

$$
w_{0}(A \vee B)=w(\langle A \vee B\rangle)=w(\langle A\rangle \vee\langle B\rangle)=w_{0}(A)+w_{0}(B) .
$$

Observe that the state $w_{0}$ induced by $w$ vanishes identically on $P(S)$ and $\hat{w}_{0}=w$. Finally, it is not difficult to check that ${ }^{\wedge}: s \mapsto \hat{s}$ is continuous and that the inverse mapping is too. Hence ${ }^{\wedge}: s \mapsto \hat{s}$ is an affine homeomorphism between the two statespaces.

We recall that in [3], it was proved that if $S$ is an incomplete inner product space and $F(S)$ admits a state, then this has to be identically zero on $P(S)$ (i.e. $\mathcal{S}(F(S))=\mathcal{S}_{0}(F(S))$ ). As a consequence of this, Theorem 1 and Proposition 5, we have the following theorem that describes the state-space of $F(S)$ for the case in which $S$ is an incomplete strongly dense inner product space. (The state-space of $F(S)$ for the case in which $S$ is a complete inner product space was first described in [1].)

THEOREM 2. Let $S$ be a strongly dense, incomplete inner product space. There is an affine homeomorphism $\phi: s \mapsto s^{\phi}$ between the state-space of $F(S)$ and the face $\mathcal{S}_{0}(F(\bar{S}))$ consisting of all the states that vanish identically on $P(\bar{S})$. Each state $s$ on $F(S)$ is the restriction of $s^{\phi}$, i.e. $s(M)=s^{\phi}(\bar{M})$, for all $M \in F(S)$. 
We remark that we do not know of an example of an incomplete inner product space $S$, satisfying $\mathcal{S}(F(S))=\emptyset$.

If $H$ is an infinite dimensional complex Hilbert space, $\mathcal{B}(H)$ the $C^{*}$-algebra of bounded operators on $H$ and $\mathcal{C}(H)$ the closed two-sided ideal of compact operators on $H$, we can form the quotient $\mathcal{B}(H) / \mathcal{C}(H)$, which is a $C^{*}$-algebra, the Calkin algebra (see [11]). It is clear that $F(H)$ can be identified with the set of projections in $\mathcal{B}(H)$. As a consequence of the Gleason-Christenson-Yeadon theorem (see [8], [5], [16] and [17]) and the Spectral Mapping theorem, it follows that the state-space of $F(H)$ is affinely homeomorphic to the state-space of $\mathcal{B}(H)$. Moreover, since every self-adjoint compact operator is the norm limit of operators with finite rank, $\mathcal{S}_{0}(F(H))$ is affinely homeomorphic to the state-space of the Calkin algebra $\mathcal{B}(H) / \mathcal{C}(H)$. Thus, we have the following corollary. (The correspondence between pure states and maximal left ideals for $C^{*}$-algebras is well known [11, Theorem 10.2.10].)

COROLlaRY 1. Let $S$ be a strongly dense incomplete complex inner product space. There is an affine homeomorphism $\psi: s \mapsto s^{\psi}$ between the state-space of $F(S)$ and the state-space of the Calkin algebra $\mathcal{B}(H) / \mathcal{C}(H)$, where $H=\bar{S}$. Each state $s$ on $F(S)$ is the restriction of $s^{\psi}$; i.e. $s(M)=s^{\psi}\left(P_{\bar{M}} / \mathcal{C}(H)\right)$, for all $M \in F(S)$. In view of this homeomorphism, we can also characterize the pure states in $\mathcal{S}(F(S))$ as follows. There is a one to one correspondence between the pure states on $F(S)$ and the maximal left ideals of $\mathcal{B}(H)$ containing $\mathcal{C}(H)$.

We conclude with the following remark.

REMARK 1. For an inner product space $S$, we can consider the orthomodular poset of all splitting subspaces of $S$; i.e. the system of subspaces $M \subset S$ satisfying $M \oplus M^{\perp}=S$. This is denoted by $E(S)$ and is always contained in $F(S)$. It is possible to factorize $E(S)$ in exactly the same manner as it was done with $F(S)$, and the resultant quotient, say $\mathfrak{E}(S)$, will be an orthomodular poset. However, $\mathfrak{E}(S)$ can be very 'poor' - even for strongly dense spaces. Indeed, in [14], a hyperplane $S_{0}$ of a separable Hilbert space was constructed such that $E\left(S_{0}\right)$ consists merely of the finite/co-finite dimensional subspaces of $S_{0}$. Thus, for this hyperplane, $\mathfrak{F}\left(S_{0}\right) \cong \mathfrak{F}\left(\overline{S_{0}}\right)$, whereas $\mathfrak{E}(S)=$ $\left\{\langle\{0\}\rangle,\left\langle S_{0}\right\rangle\right\}$, and therefore, a similar conclusion to Theorem 2 cannot be drawn if $F(S)$ is interchanged with $E(S)$.

\section{REFERENCES}

1. J. F. Aarnes, Quasi-states on $C^{*}$-algebras, Trans. Amer. Math. Soc. 149 (1970), 601-625.

2. I. Amemiya and H. Araki, A remark on Piron's paper, Publ. Res. Inst. Math. Sci., Kyoto Univ., Ser. A 2 (1966-1967), 423-427.

3. E. Chetcuti and A. Dvurečenskij, A finitely additive state criterion for the completeness of inner product spaces, Lett. Math. Phys. 64 (2003), 221-227.

4. E. Chetcuti and A. Dvurečenskij, The existence of finitely additive states on orthogonally closed subspaces of incomplete inner product spaces, Lett. Math. Phys. 67 (2004), 75-80.

5. E. Christensen, Measures on projections and physical states, Comm. Math. Phys. 86 (1982), 529-538.

6. A. Dvurečenskij, Gleason's theorem and its applications (Kluwer Acad. Publ., Dordrecht, Ister Science Press, Bratislava, 1992).

7. A. Dvurečenskij, T. Neubrunn and S. Pulmannová, Finitely additive states and completeness of inner product spaces, Found. Phys. 20 (1990), 1091-1102. 
8. A. M. Gleason, Measures on the closed subspaces of a Hilbert space, J. Math. Mech. 6 (1957), 885-893.

9. J. Hamhalter, Quantum measure theory (Kluwer Acad. Publ., Dordrecht, 2003).

10. J. Hamhalter and P. Pták, A completeness criterion for inner product spaces, Bull. London Math. Soc. 19 (1987), 259-263.

11. R. V. Kadison and J. R. Ringrose, Fundamentals of the theory of operator algebras I,II (Academic Press, Inc., 1986).

12. F. Maeda and S. Maeda, Theory of symmetric lattices (Springer-Verlag, 1970).

13. P. Pták, FAT - CAT (in the state space of quantum logics), in Proceedings of "Winter School of Measure Theory", Liptovský, Ján 1988 (Czechoslovakia), 113-118.

14. P. Pták and H. Weber, Lattice properties of subspace families in an inner product space, Proc. Amer. Math. Soc. 129 (2001), 2111-2117.

15. V. S. Varadarajan, Geometry of quantum theory (Springer-Verlag, 1985).

16. F. J. Yeadon, Measures on projections in $W^{*}$-algebras of type II, Bull. London. Math. Soc. 15 (1983), 139-145.

17. F. J. Yeadon, Finitely additive measures on projections in finite $W^{*}$-algebras, Bull. London. Math. Soc. 16 (1984), 145-150. 